\title{
ВMJ Global Health Event-based surveillance at health facility and community level in low- income and middle-income countries: a systematic review
}

To cite: Kuehne A, Keating $P$, Polonsky J, et al. Event-based surveillance at health facility and community level in lowincome and middle-income countries: a systematic review. BMJ Global Health 2019;4:e001878. doi:10.1136/ bmjgh-2019-001878

Handling editor Seye Abimbola

Received 30 July 2019

Revised 26 August 2019 Accepted 30 August 2019

Check for updates

(C) Author(s) (or their employer(s)) 2019. Re-use permitted under CC BY-NC. No commercial re-use. See rights and permissions. Published by BMJ.

${ }^{1}$ UK Public Health Rapid Support Team, London, United Kingdom ${ }^{2}$ Public Health England, London, United Kingdom

${ }^{3}$ Department of Infectious Disease Epidemiogy, London School of Hygiene and Tropical Medicine, London, United Kingdom

${ }^{4}$ Department of Health Emergency Information and Risk Assessment, World Health Organization, Geneva, Switzerland

${ }^{5}$ Faculty of Medicine, University of Geneva, Geneva, Switzerland

Correspondence to Dr Anna Kuehne; anna.kuehne@Ishtm.ac.uk

\section{ABSTRACT}

Background The International Health Regulations require member states to establish "capacity to detect, assess, notify and report events". Event-based surveillance (EBS) can contribute to rapid detection of acute public health events. This is particularly relevant in low-income and middleincome countries (LMICs) which may have poor public health infrastructure. To identify best practices, we reviewed the literature on the implementation of EBS in LMICs to describe EBS structures and to evaluate EBS systems.

Methods We conducted a systematic literature search of six databases to identify articles that evaluated EBS in LMICs and additionally searched for grey literature. We used a framework approach to facilitate qualitative data synthesis and exploration of patterns across and within articles.

Results We identified 778 records, of which we included 15 studies concerning 13 different EBS systems. The 13 EBS systems were set up as community-based surveillance, health facility-based surveillance or open surveillance (ie, notification by non-defined individuals and institutions). Four systems were set up in outbreak settings and nine outside outbreaks. All EBS systems were integrated into existing routine surveillance systems and pre-existing response structures to some extent. EBS was described as useful in detecting a large scope of events, reaching remote areas and guiding outbreak response. Conclusion Health facility and community-based EBS provide valuable information that can strengthen the early warning function of national surveillance systems. Integration into existing early warning and response systems was described as key to generate data for action and to facilitate rapid verification and response. Priority in its implementation should be given to settings that would particularly benefit from EBS strengths. This includes areas most prone to outbreaks and where traditional 'routine' surveillance is suboptimal.

\section{BACKGROUND}

The International Health Regulations (IHR) require member states to have "the capacity to detect, assess, notify and report events". ${ }^{1}$ An event is defined as "a manifestation of disease

\section{Key questions}

\section{What is already known?}

- Event-based surveillance (EBS) aims to contribute to rapid detection of acute public health events, and supplements indicator-based surveillance (IBS), the backbone of national surveillance systems.

- To date, no assessment of evidence for best practices exists.

What are the new findings?

- The scope of EBS is broader than IBS in several aspects: (1) In outbreaks, EBS was able to identify outbreak-disease cases and programming gaps helping to guide the response; (2) EBS was able to rapidly identify outbreaks and other health hazards that were not detected by IBS, or before they were detected by IBS; (3) EBS was able to reach remote areas and was most used where health facilities were scarce.

- Good communication with communities and othe stakeholders were described as key; in addition, linkage to IBS surveillance and rapid response was described as essential for all systems as every verified event needs an assessment and potentially a response.

What do the new findings imply?

- EBS is a valuable source of data that can strengthen the early warning function of national surveillance systems, if integrated into existing surveillance and linked to response structures.

- Priority in the implementation of EBS should be given to settings that would particularly benefit from EBS' strengths such as areas most prone to outbreaks, where access to the formal health system is limited, where traditional 'routine' surveillance is suboptimal and where timeliness of notification of epidemicprone diseases is of particular importance to mounting a rapid response.

or an occurrence that creates a potential for disease". Event-based surveillance (EBS) is the organised collection, monitoring, assessment 
and interpretation of mainly unstructured, ad hoc information regarding health events or risks, which may represent an acute risk to human health. ${ }^{2}$ EBS should be monitored and responded to in real-time. EBS, alongside indicatorbased surveillance (IBS), is a component of the early warning, alert and response (EWAR) function of a national surveillance system. ${ }^{2}$ The objective of EWAR is to support the early detection of, and rapid response to, acute public health events of any origin. ${ }^{2}$ Thus, EWAR is a key function of surveillance, particularly in low-income and middleincome country (LMIC) settings and for humanitarian emergencies where the epidemic risk is high and other elements of the surveillance architecture may be limited or absent. ${ }^{2}$ EBS may provide the earliest detection of any acute public health event, particularly those that are not well detected by IBS, or not detected rapidly enough.

Guidance documents discuss the implementation of EBS within national surveillance systems, prior to and during humanitarian emergencies. ${ }^{2-6}$ However, to date no assessment of evidence for best practices exists. We evaluated the peer-reviewed and grey literature relating to the implementation of EBS for outbreak detection in
LMICs in both emergency and non-emergency settings in order to provide a systematic description of EBS and to evaluate the strengths and weaknesses of EBS across both contexts.

\section{METHODS \\ Key outcomes}

The key outcomes included summaries of the (1) quality of available evaluations, (2) EBS system structure and event definitions in use, and (3) evaluations of surveillance attributes of the EBS systems.

\section{Search strategy}

Following PRISMA guidelines, we conducted a systematic review to identify peer-reviewed literature that presented data collected by EBS systems in LMICs and/or evaluated EBS systems in LMICs. We searched six databases: MEDLINE (Ovid MEDLINE and Epub Ahead of Print, In-Process \& Other Non-Indexed Citations and Daily), EMBASE, Global Health, Northern Light Life Sciences Conference Abstracts, CINAHL Plus with full text

Table 1 Systematic and grey literature review on event-based surveillance in the field: definitions

EBS For the purpose of this review, we defined EBS as "the organised collection, monitoring, assessment and interpretation of information of mainly unstructured ad hoc information regarding health events or risks, which may represent an acute risk to human health"2 and require rapid reporting and assessment. ${ }^{23}$ The events can relate to the occurrence of epidemic-prone diseases and other chemical and environmental hazards in humans (eg, cluster of cases of disease, unusual disease patterns, unexplained deaths, chemical spills) or related to potential exposure for humans (eg, mass deaths among animals). ${ }^{2} 3$

We included EBS systems (as distinct from IBS ${ }^{2}$ ) characterised by the following attributes: (1) the source of signal can be a report from the general public, healthcare workers, community-level volunteers and it is not limited to the use of data and other information that arrive via a formal IBS system; (2) notifiable events are not defined by symptoms or syndromes but by (specified or unspecified) events related to health threats; (3) notification from the field to the EBS system is immediate (rather than planned weekly or monthly); (4) every notified signal requires rapid verification; (5) every verified event requires a timely response.

EBS as defined by $\mathrm{WHO}^{2}$ can include an epidemic intelligence component that is based on the systematic automated analysis of various media sources (such as Epidemic Intelligence from Open Sources ${ }^{29}$ and the Global Public Health Intelligence Network ${ }^{30}$ on global scale). However, given the difference in structure and remit, we restricted our review to field-based EBS sourced from informants in health facilities or in the community.

\begin{tabular}{|c|c|}
\hline Signal & $\begin{array}{l}\text { A signal is reported data or information which represent a potential acute risk to human health. It is transmitted } \\
\text { immediately and has not yet been verified to meet the event definition of the EBS system. }{ }^{2}\end{array}$ \\
\hline Event & $\begin{array}{l}\text { The IHR define an event as "(...)a manifestation of disease or an occurrence that creates a potential for disease; } \\
\text { (...)". } .^{1} \text { In the context of event-based surveillance, an 'event' refers to 'a signal' that has been verified to meet the } \\
\text { event definition of the EBS system. }\end{array}$ \\
\hline Alert & $\begin{array}{l}\text { In this document (as in the WHO guidance } 2014^{2} \text { ), "an alert will refer to a public health event that has been (i) verified } \\
\text { and (ii) risk assessed and (iii) requires an intervention (an investigation, a response or a communication)." } 2\end{array}$ \\
\hline $\begin{array}{l}\text { Community-based } \\
\text { surveillance (CBS) }\end{array}$ & $\begin{array}{l}\text { "CBS is the systematic detection and reporting of events of public health significance within a community } \\
\text { by community members". }{ }^{11} \text { It is a surveillance system (IBS or EBS) that relies on defined individuals from the } \\
\text { community (often called community volunteers) who received special training on event definition (for EBS) or case } \\
\text { definitions (for IBS) and who notify signals to the surveillance system. }\end{array}$ \\
\hline $\begin{array}{l}\text { Health facility-based } \\
\text { surveillance }\end{array}$ & $\begin{array}{l}\text { A surveillance system (IBS or EBS) that relies on health professionals notifying signals to a next level in the } \\
\text { surveillance system. }\end{array}$ \\
\hline Open surveillance & $\begin{array}{l}\text { We use the term 'open surveillance' to describe systems that did not specify who can notify signals but were open to } \\
\text { receive signals from anyone (including lay people, media, NGOs, health professionals, teachers etc). }\end{array}$ \\
\hline Outbreak setting & Refers to a setting in which EBS was implemented during an outbreak to enhance outbreak-specific surveillance. \\
\hline Routine setting & $\begin{array}{l}\text { Refers to a setting in which the purpose of the EBS system is to contribute to routine surveillance of defined or } \\
\text { undefined events to detect outbreaks and other public health emergencies outside outbreaks. }\end{array}$ \\
\hline
\end{tabular}

CBS, community-based surveillance; EBS, event-based surveillance; IBS, indicator-based surveillance; IHR, International Health Regulations; NGO, non-government organisation; WHO, World Health Organisation. 
and Africa Wide Information. We included literature published since the earliest date indexed in each database up to 20 August 2018 in English, French, German, Portuguese or Spanish. The search terms were related to (1) event-based surveillance and early warning alert and response, or (2) different types and channels of surveillance that are used to detect outbreaks or epidemics and (3) LMICs and regions (online supplementary file 1). The search strategies included indexed terms where possible and the list of LMICs was adapted from the standard list available at OVID MEDLINE based on World Bank Group classification 2017-2018. ${ }^{7}$ We adapted the search strategy for MEDLINE and tailored it to each database. Furthermore, we conducted a backward citation search and searched the references for each included article for relevant literature. We repeated all steps of the systematic assessment of titles and abstracts and full texts for all cited references.

Additional grey (unpublished) literature was retrieved by contacting epidemiologists at agencies and networks known to be involved in implementation or evaluation of EBS at the London School of Hygiene and Tropical Medicine, Médecins Sans Frontières, Norwegian Red Cross, CARE, US Centers for Disease Control (US CDC), WHO, and the Training Programs in Epidemiology and Public Health Interventions Network (TEPHINET).

\section{Definitions}

We defined the terms EBS and associated terminology in accordance with WHO guidance ${ }^{23}$ (table 1).

\section{Selection of literature}

Following the elimination of duplicates, two researchers independently assessed the potential relevance of all papers, starting with titles and abstracts (screening) and full text (data extraction) using set inclusion and exclusion criteria. A consensus was reached for any discrepancy through discussion between the two researchers. For conference abstracts that were identified during the literature search, we contacted the corresponding author to obtain additional information.

\section{Inclusion criteria}

The full text referred to EBS as defined above and to LMICs as defined by the World Bank Group 2017-2018. ${ }^{7}$

\section{Exclusion criteria}

We excluded any full texts that described the EBS system but did not provide any data (quantitative or qualitative) on its functioning or effectiveness. We excluded any full texts that referred exclusively to (1) either case-based or syndromic IBS systems, or (2) systems using thresholds (of numbers of observations) to generate an alert. We excluded full texts that described mixed surveillance systems (IBS and EBS)

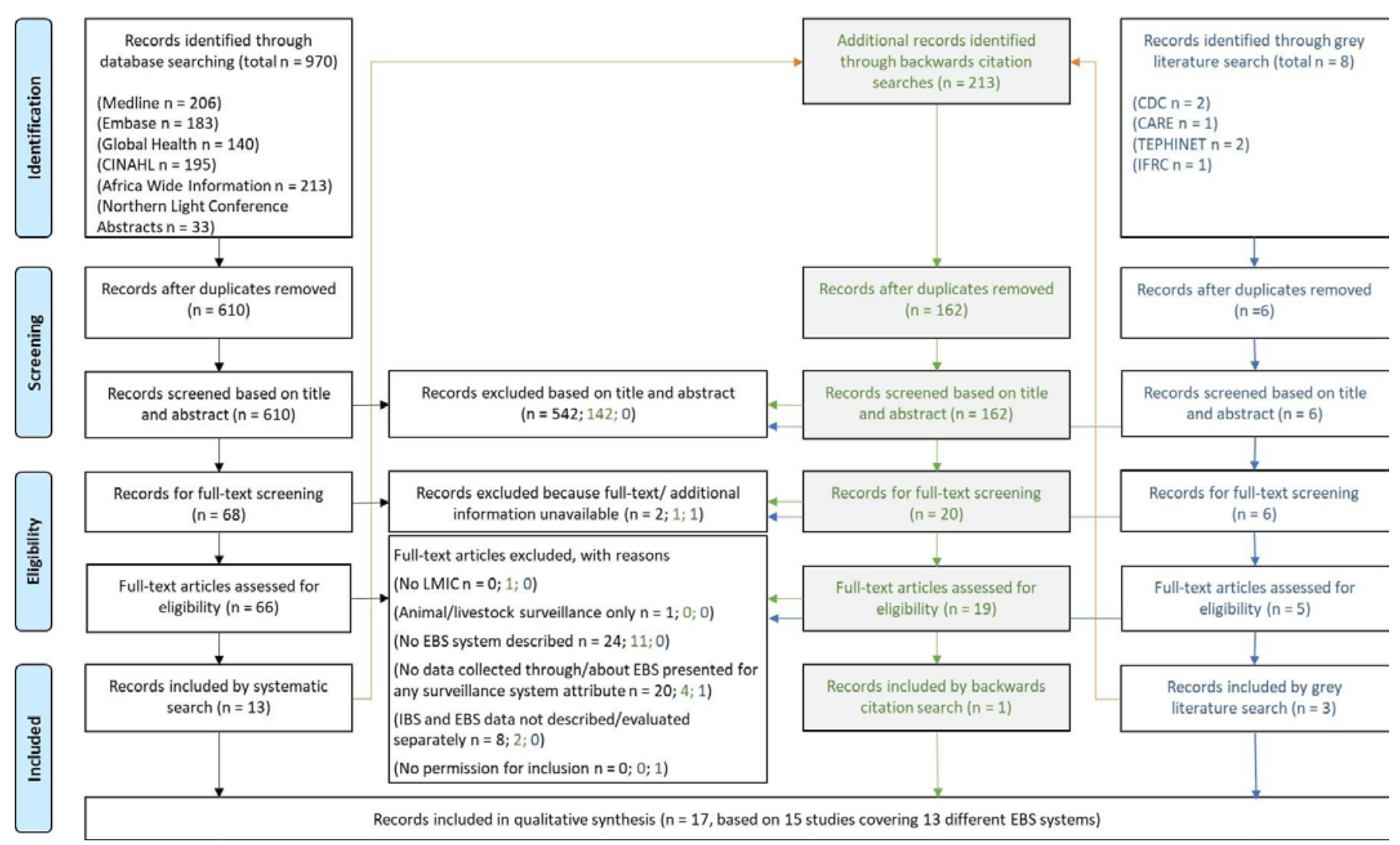

Figure 1 Systematic and grey literature review on event-based surveillance in the field: PRISMA flow chart for systematic literature review (black ink letters) and grey literature search (blue ink letters) and backward citation search (green ink letters) describing identification, screening, eligibility and inclusion. EBS, event-based surveillance; IBS, indicator-based surveillance; LMIC, low-income and middle-income countries. 


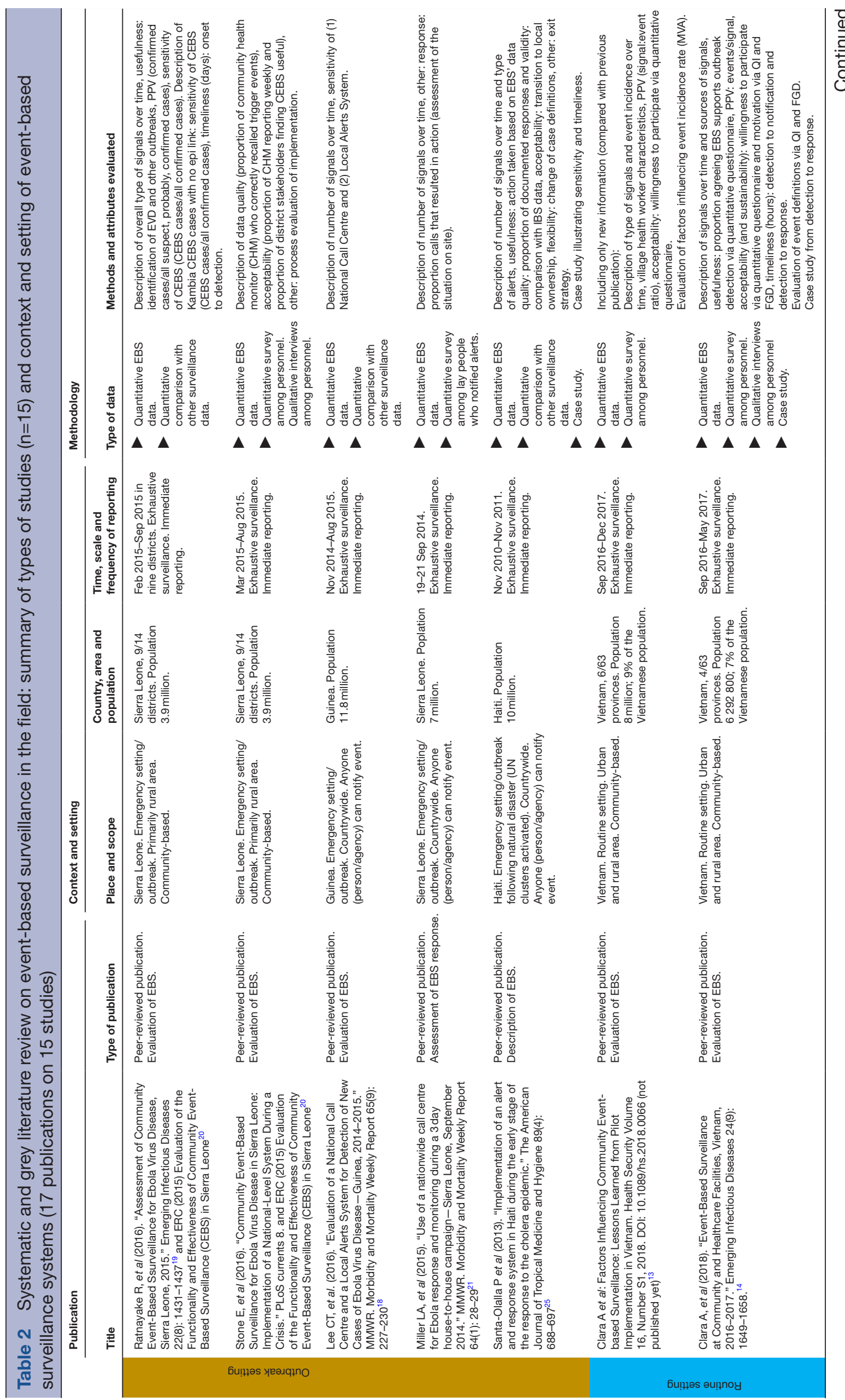




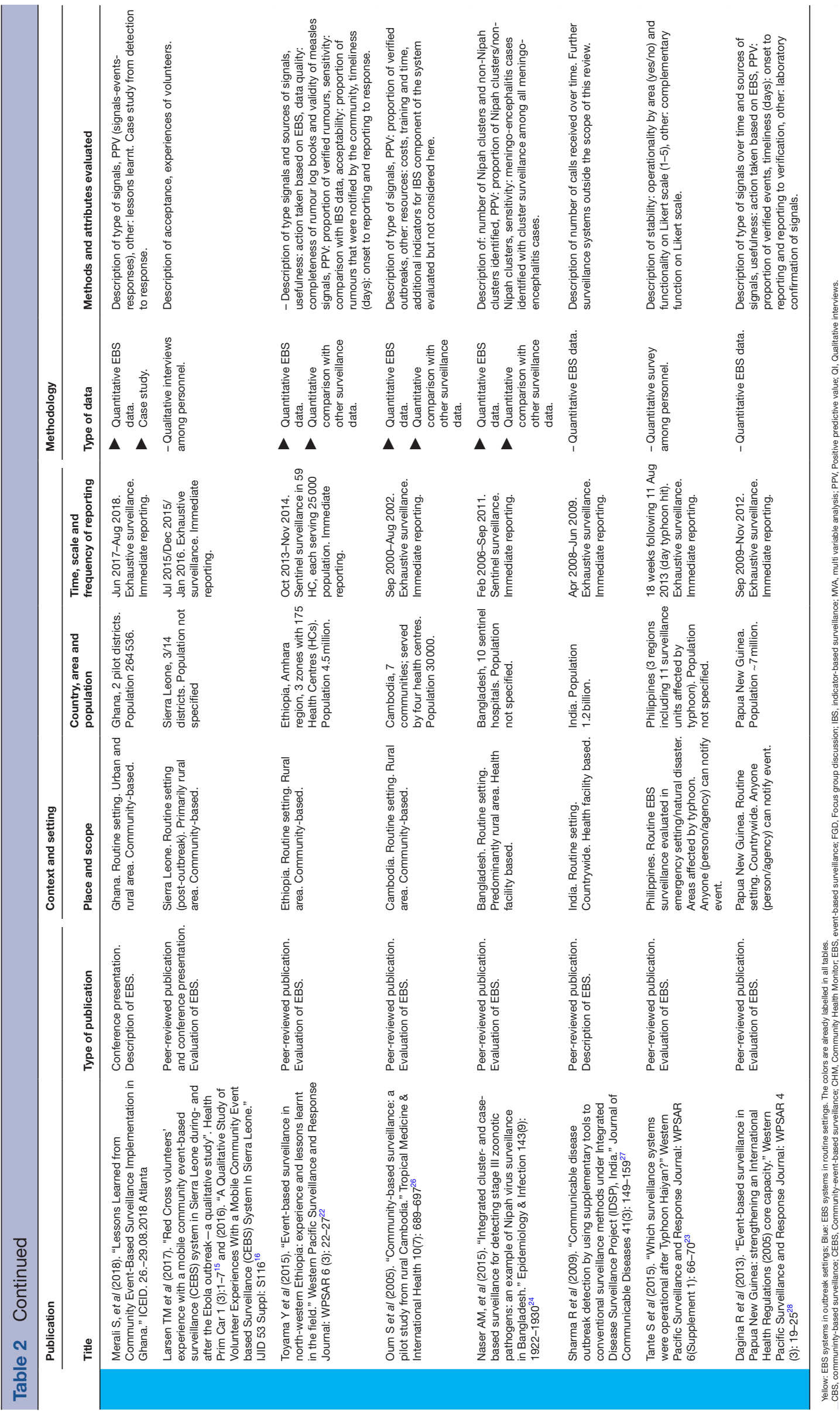


if the evaluation did not provide detailed information on the EBS component. In addition, descriptions of EBS that focused on animal health without any link to the human health were excluded. Finally, we did not include global EBS that relied on algorithms for detection of media or social media activity only.

In the case of multiple publications based on the same EBS system/dataset, the quality and content were assessed simultaneously. Structure and components are described by EBS system (and not by publication or by dataset).

\section{Assessment of quality of studies}

We assessed the quality of the evidence using the GRADE approach. ${ }^{8}$ In addition, we developed a score to evaluate the quality of the evaluation based on the guidance by CDC for evaluating surveillance systems. ${ }^{9}$ We based our approach on the eight key elements of surveillance system evaluation described by $\mathrm{CDC}^{9}$ and added two additional categories: "response mechanism described" and "feedback mechanism to stakeholders described". We scored the quality of description of the surveillance system (0-2 points). Zero points applied for "not stated/ missing information", one point for "incomplete description" and two points for "comprehensive description". We calculated a summary score (0-20 points) for the quality of description. In addition, we graded each attribute evaluated and the comprehensiveness of evaluation using a simple colour scheme (green: comprehensively evaluated; yellow: partly evaluated; red: not evaluated).

\section{Data extraction}

We extracted information for each study using a standardised form assessing quality of the description, context, event definitions, characteristics and attributes evaluated.

\section{Data synthesis}

We used a framework approach ${ }^{10}$ to facilitate qualitative data synthesis and exploration of patterns within and across articles. We summarised quantitative results without pooling the data as the lack of standardisation between surveillance systems and evaluations does not allow for the calculation of quantitative summary measures. Following an inductive process, we developed typologies of EBS systems separately for outbreak and routine contexts. We synthesised available information for each of 10 defined attributes of surveillance systems ${ }^{9}$ using the framework approach. ${ }^{10}$

\section{Patient involvement}

Design, literature search, data synthesis and interpretation were done without patient involvement.

\section{RESULTS}

\section{Selection of literature}

After deduplication, we screened titles and abstracts of 778 records (systematic literature search, grey search and backwards search combined) (figure 1). We screened full texts for 90 records, of which we included 17 (figure 1).
Among the 17 included records were 13 peer-reviewed publications, one manuscript under peer review, two conference abstracts including presentations and one agency report.

All full texts assessed and the reason for exclusion are available in online supplementary file 2.

\section{Quality assessment}

The 17 identified records referred to 15 studies (based on individual sets of data) and 13 different EBS systems. ${ }^{8-24}$ For the quality appraisal, we assessed each of the 15 studies. Three studies were of low evidence (observational studies with comparison groups) and 12 were of very low evidence (observational studies without any comparison group), according to GRADE.

The quality of description of the EBS system varied from a summary score of 5/20 to 20/20 (online supplementary file 3). All 15 studies described the objective of the surveillance system, the event(s) under surveillance and the components of the EBS system to some extent (online supplementary file 3 ). With regards to the attributes described, 9 of the 17 studies described the positive predictive value of signals to events for the EBS system to some extent. All other attributes were rarely described (online supplementary file 3 ).

\section{Data extraction}

Type of studies and context of EBS systems

Of the 15 included studies, 11 studies were evaluations of EBS systems, three were descriptions of EBS systems and one was an assessment of the response to EBS alerts (table 2).

\section{Structure, components and functioning of EBS systems}

Event definitions were disease specific in the four EBS systems in outbreak settings and one routine EBS system. In other routine surveillance systems, definitions were a mix of events targeting emerging diseases, unusual events and early detection of outbreaks (table 3).

Trained lay community volunteers (CVs) notified events for 6/13 EBS systems, healthcare workers notified events in 2/13 EBS systems, and any member of the public was able to notify events using the two public telephone hotlines and in the three centralised surveillance systems (table 4). A verification and risk assessment mechanism was described for 11/13 EBS systems and a standardised algorithm for verification was mentioned in 3/13 EBS systems (table 4). All EBS systems were integrated into the routine surveillance system to some extent (table 4). Routine surveillance staff were involved in the response in all systems that specified the responding body $(12 / 13)$. Six of 13 EBS systems specified a feedback mechanism to stakeholders (table 4 ).

\section{Data synthesis}

Types of EBS systems in outbreak contexts Community-based surveillance in outbreak contexts

We identified one community event-based surveillance (CEBS) system operating during an outbreak: the Sierra 


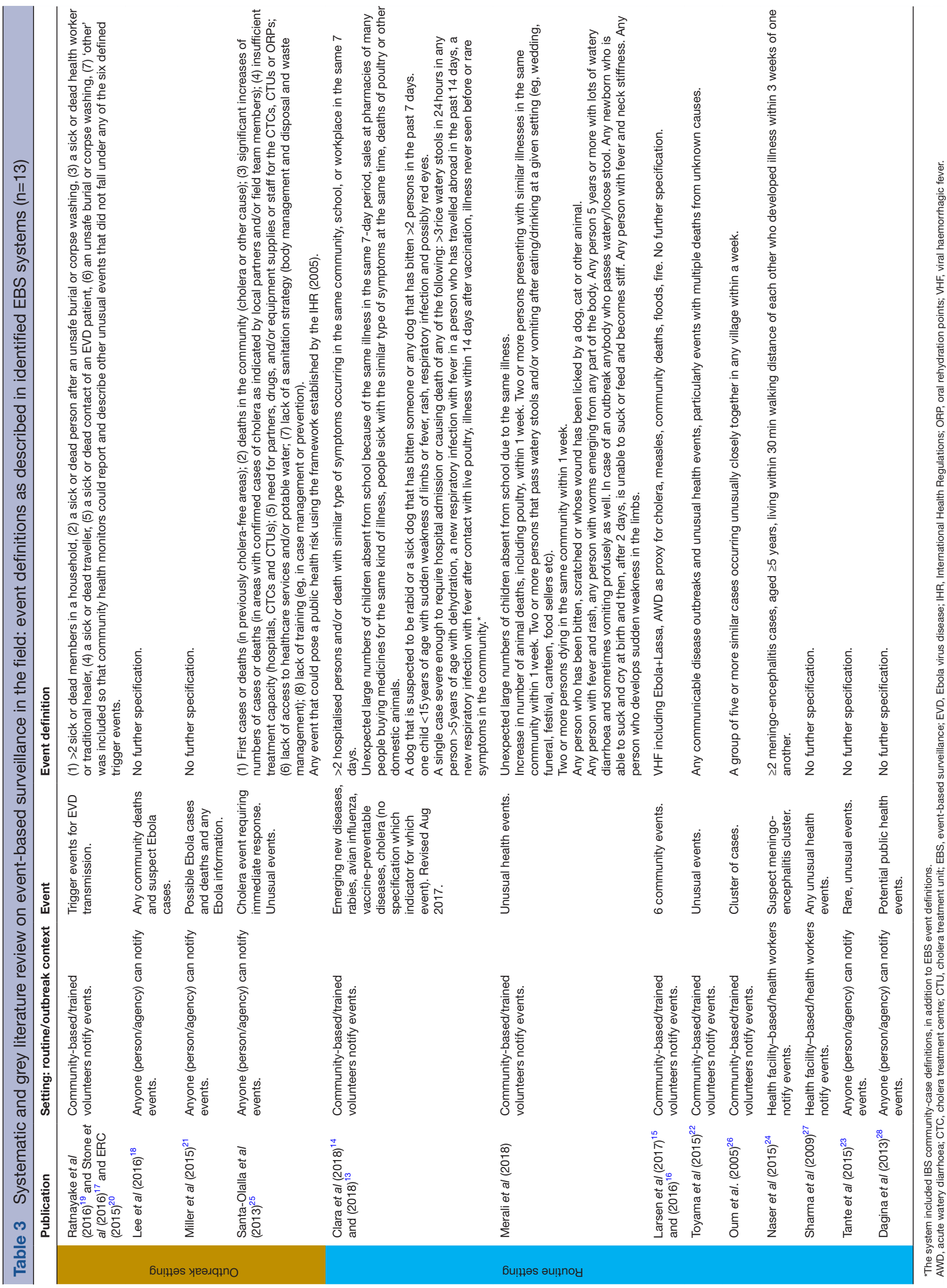


Leonean CEBS implemented during the Ebola outbreak at national level. ${ }^{17} 1920 \mathrm{CVs}$ and their supervisors were specifically identified and rapidly trained; supervisory staff provided verification and assessment. The districtlevel Ebola response system that was already in place at the local level conducted follow-up if needed. ${ }^{17} 1920 \mathrm{CVs}$ notified defined Ebola-related events (table 4). ${ }^{1719}{ }^{20}$ No verification or assessment algorithm was specified. ${ }^{19}$

\section{Open surveillance}

We identified three EBS systems that were 'open' to receive outbreak-specific event notifications from anyone: the telephone hotlines during the Ebola outbreak in Guinea $^{18}$ and Sierra Leone ${ }^{21}$ allowed anyone to notify unspecified Ebola-related events; signals received by call centres were forwarded to the Ebola response structure in place for verification and response. ${ }^{1821}$ Similarly, the EBS system in place in Haiti at the start of the cholera outbreak allowed partners within and outside the UN health cluster to notify an additional list of defined cholera-related events, including the lack of access to healthcare and sanitation. ${ }^{25}$ The focus was expanded to unusual events in general after the outbreak. ${ }^{25}$ Notifications were received at central level; verification, assessment and response processes were co-ordinated centrally but supported locally by field response teams. The assessment consisted of several standard items to answer. ${ }^{25}$

Types of EBS systems in routine contexts Community-based surveillance in routine contexts

We identified five CBS systems that were functioning as routine EBS systems in Vietnam, ${ }^{13}{ }^{14}$ Ghana, ${ }^{12}$ Ethiopia, ${ }^{22}$ Cambodia ${ }^{26}$ and Sierra Leone. ${ }^{1516} \mathrm{CV}$ s were already in place before the implementation of all five systems. ${ }^{10121319-22} \mathrm{CVs}$ notified defined syndromes to IBS in addition to events to EBS in four systems. ${ }^{12-16}{ }^{26} \mathrm{CV}$ s working in EBS-only systems were instructed to report any outbreak or unusual event ${ }^{22}$; CVs working in combined IBS and EBS systems had specific definitions for the events (table 4). ${ }^{12-1626}$ All EBS systems in communities were built on existing IBS systems and infrastructure. No verification or assessment algorithm was specified for any of the systems.

\section{Health facility-based surveillance in routine contexts}

We identified two different types of health facility-based surveillance: in Bangladesh, routine health facility-based IBS in a set of hospitals was complemented by EBS in a way that clusters of meningo-encephalitis cases were notified immediately and investigated to identify small-area Nipah outbreaks. ${ }^{24}$

In India, a hotline was set up to notify unusual events that was available to health professionals. ${ }^{27}$ No verification or assessment algorithm was specified for any of the systems.

\section{Open surveillance in routine contexts}

We identified two 'open' surveillance systems: the EBS system in the Philippines after Typhoon Haiyan could receive event information through routine surveillance channels or informal reporting by anyone; steps for verification, assessment and response were not specified in the paper. ${ }^{23}$ In Papua New Guinea (PNG), anyone could notify events to a national EBS coordinator, who reached out to provincial health authorities for verification, assessment and response. A standardised verification and assessment form was used and kept as a log. ${ }^{28}$

\section{Surveillance attributes of EBS systems}

Data extraction for each surveillance system attribute can be found in online supplementary file 4 .

\section{Usefulness: events reported and actions taken}

All EBS systems detected local outbreaks. All nondisease-specific and disease-specific EBS systems reported to have identified confirmed cases and outbreaks among humans (eg, measles, acute respiratory infections, chickenpox, acute watery diarrhoea, malaria, whooping cough, meningitis, acute flaccid paralysis) and among animals (eg, poultry die-off, rabies, anthrax) (online supplementary file 4). ${ }^{12-15} 22$ 25-28 In addition, natural and man-made events (eg, floods, riots, chemical events, nutritional crisis) were reported. ${ }^{222528}$

In outbreak contexts, community event-based surveillance (CEBS) during the Ebola outbreak in Sierra Leone generated a large number of signals $(>90 \%)$ that were mainly reported in the category 'other' (rather than in any defined category) and the majority $(>85 \%)$ concerned deaths in the community. ${ }^{17} 1920$ Alerts were investigated by response teams that were in place. ${ }^{19}$

Open Ebola telephone hotlines during the Ebola outbreak in Sierra Leone and Guinea received signals for cases: during a 3-day campaign in Sierra Leone, $75 \%$ of calls were verified as alerts, ${ }^{21}$ while only $12 \%$ of calls were verified as alerts over 10 months in Guinea. ${ }^{18}$ Alerts were investigated by the response teams in place though the number of true events (identified case(s) meeting the case definition) was not reported.

In the open cholera EBS system in Haiti, $90 \%$ of the signals received related to cholera (increase in cases, deaths, lack of supplies etc) but also relevant non-cholera signals were received including rabies, acute flaccid paralysis, anthrax and non-infectious hazards. ${ }^{25}$ Alerts were of use to indicate underserved geographical areas within which to set up treatment centres, investigate outbreaks, and carry out community mobilisation and water and sanitation activities ${ }^{25}$ (online supplementary file 4).

In routine contexts, CEBS systems identified human and animal health events and other hazards: CEBS in Vietnam identified mostly signals of hand-foot-mouth disease, suspect dengue, chickenpox, suspect mumps, acute respiratory infections, foodborne diseases and avian influenza in poultry. ${ }^{13}$ CEBS in Ghana identified increases in animal deaths, vaccine-preventable diseases and foodborne diseases. ${ }^{12}$ CEBS in Ethiopia identified signals for outbreaks of measles, suspect rabies, anthrax, whooping cough, acute flaccid paralysis, neonatal tetanus, meningitis, acute watery diarrhoea, flood and malnutrition ${ }^{22} ; 67 \%$ of signals were verified and resulted in response, such as case 


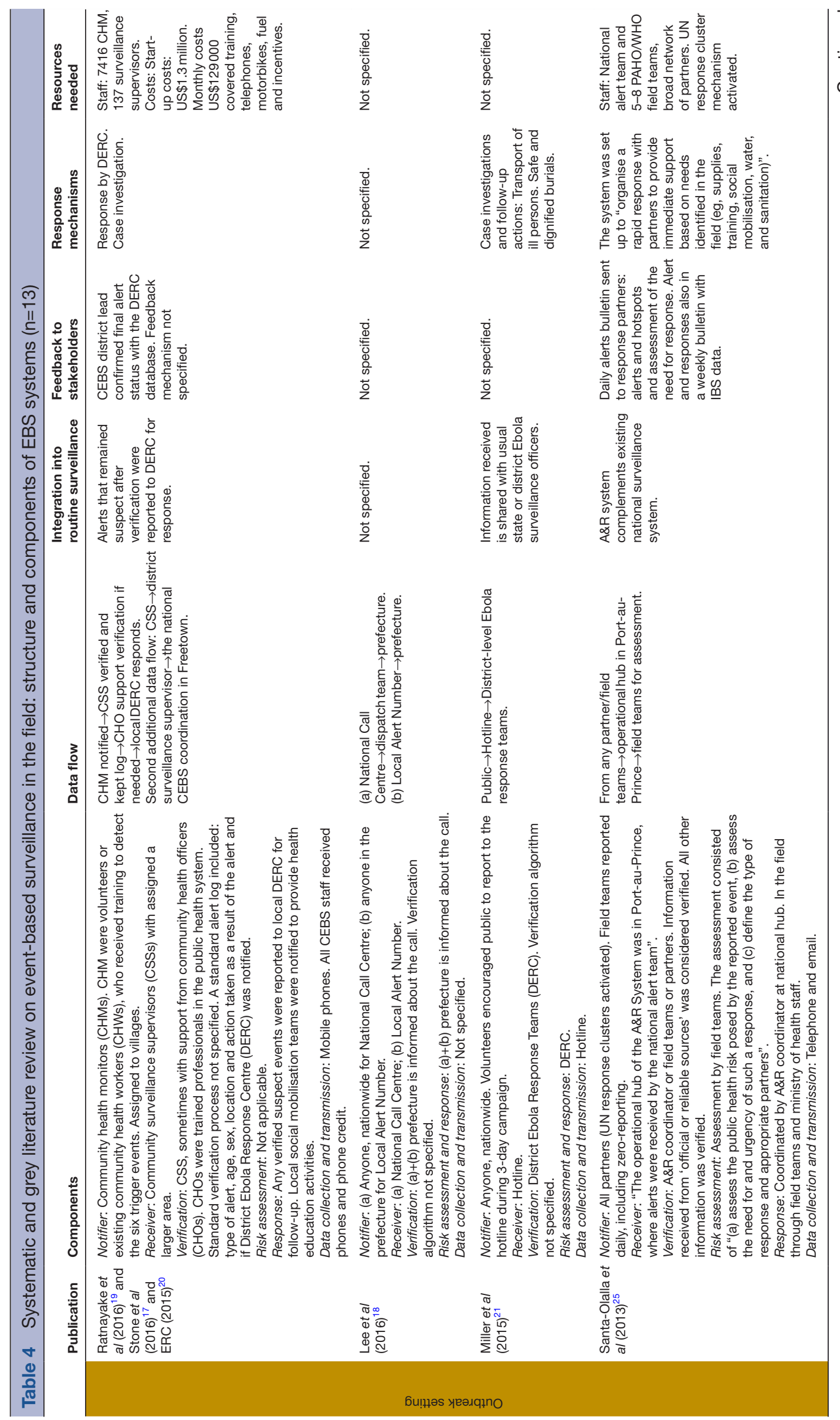




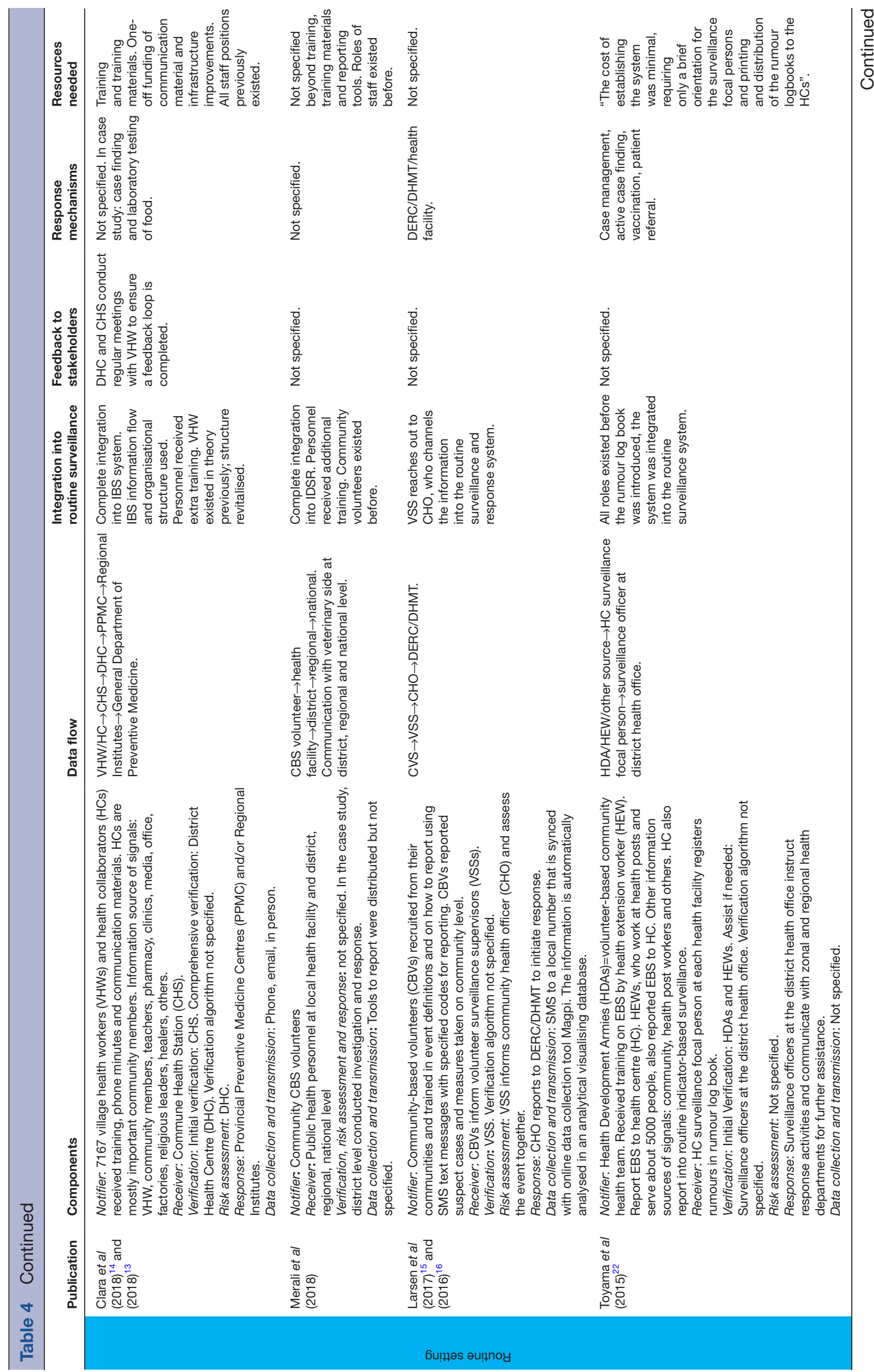




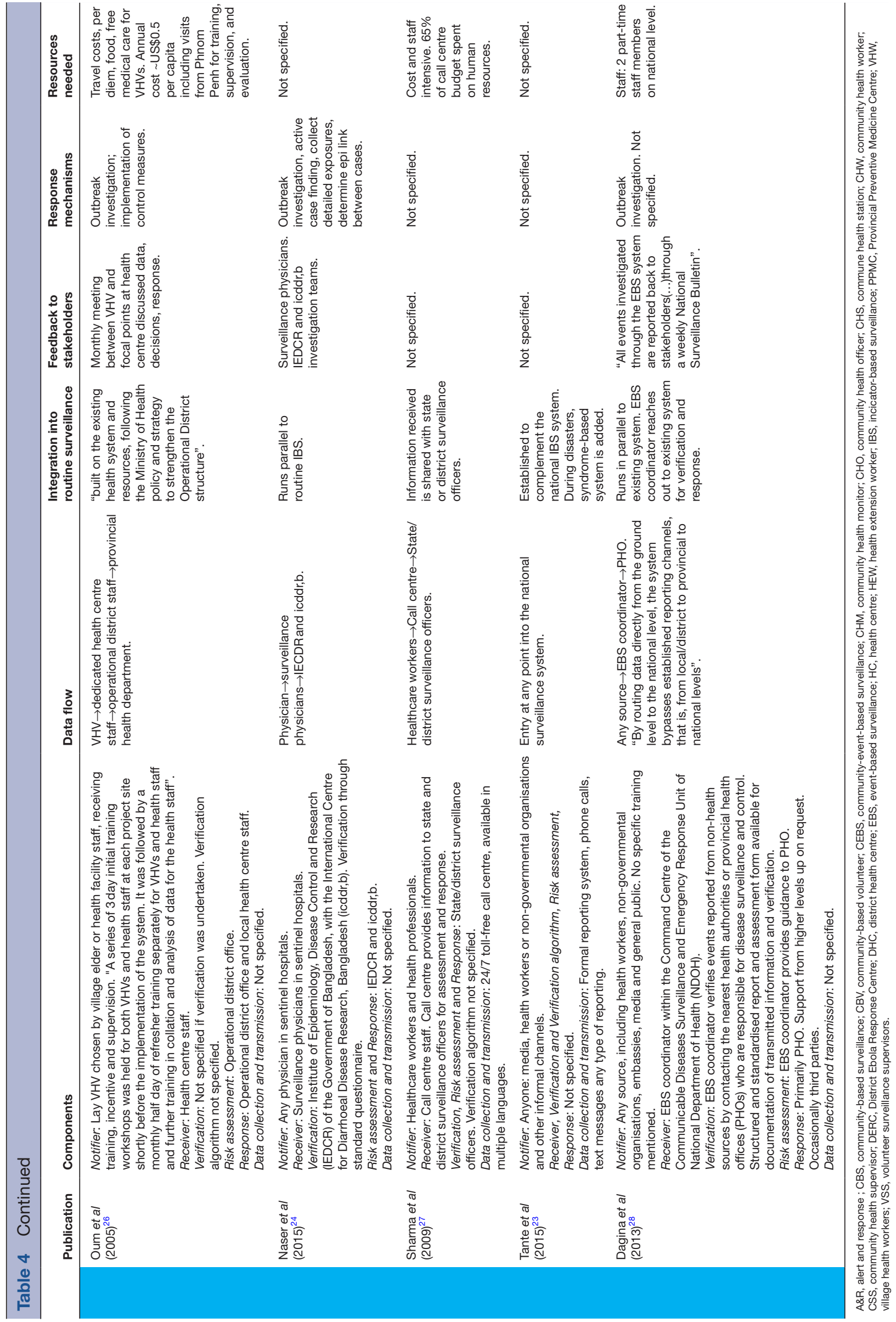


management, active case finding, patient referrals and vaccinations. ${ }^{22}$ In Cambodia, CEBS identified outbreaks of malaria, acute watery diarrhoea, measles and viral haemorrhagic fever. ${ }^{26}$

Health facility-based EBS in Bangladesh for meningoencephalitis clusters identified 62 signals that were investigated to identify chains of transmission. ${ }^{24}$ The telephone hotline for health professionals in India identified nine outbreaks but was used for non-health-related calls more than $95 \%$ of the time. ${ }^{27}$

The open generic EBS system in PNG received 61 signals from provincial health authorities, health professionals and media, NGOs and communities notified signals related to acute watery diarrhoea, influenza-like illness, acute gastrointestinal syndromes, acute fever and rash, acute neurological syndromes, acute febrile illness and other respiratory diseases. ${ }^{28}$ Alerts were investigated by provincial health authorities with support from higher levels $^{28}$ (online supplementary file 4 ).

\section{Data quality (validity and completeness)}

In the context of outbreaks, for CEBS in Sierra Leone, five of six Ebola-related trigger events were correctly recalled by less than two-thirds of CVs 3 months after implementation. ${ }^{17}$

Santa-Olalla et $a l^{25}$ compared trends of EBS signals for cholera-related events with IBS data for validity of data and saw comparable trends with regards to time and geographical distribution with the exception that more EBS signals came from hard-to-reach areas with the lowest number of partners ${ }^{25}$ (online supplementary file 4).

In a routine context in Ethiopia, similar trends in EBS signals about measles rumours and IBS measles data were reported over time. ${ }^{22}$ With regards to data completeness, $23 \%$ of signals had no records of verification and $29 \%$ had complete information on reporting and response times $^{22}$ (online supplementary file 4 ).

\section{Positive predictive value (PPV) for true events}

The interpretation of PPV across EBS systems is dependent on the types of events used for the calculation. In the context of the Ebola outbreak, 12126 signals were received through CEBS for Ebola in Sierra Leone, of which 287 were ultimately confirmed to meet the suspected, probable or confirmed case definition (PPV $0.024,287 / 12$ 2126) and 16 Ebola cases were confirmed ${ }^{19}$ (online supplementary file 4 ).

In the routine surveillance context of Vietnam, an overall PPV of 0.07 (176 events/2520 signals) for CEBS was reported based on a list of defined events. PPV increased significantly over time after evaluation visits and improved event definitions from 0.06 initially to $0.12 .{ }^{13}$ CEBS in Ghana, Ethiopia and Cambodia reported PPVs of $>0.60$ for the signals to be an event ${ }^{12226}$ (online supplementary file 4 ).

The EBS telephone hotline in India received 44484 calls from health professionals, of which 1185 were health related, 112 were outbreak signals and 9 were true outbreak alerts (PPV for a signal to be an alert 0.08; 9/112). ${ }^{27}$ The PPV for meningo-encephalitis cluster reported by health professionals to be a true Nipah cluster was 0.17 (10 clusters/62 signals). ${ }^{15}$

In PNG's centralised routine EBS system, of 61 signals, 51 proved to be true events (PPV 0.84) ${ }^{28}$ (online supplementary file 4 ).

\section{Sensitivity}

In outbreak context, Ratnayake $e t a l^{19}$ reported that $30 \%$ $(16 / 53)$ of all confirmed cases of Ebola in the area where CEBS for Ebola was implemented were identified by CEBS. In Guinea, 3.9\% (71/1838) of all confirmed Ebola cases were identified by signals received at the national Ebola call centre and 54\% (120/221) of all confirmed Ebola cases were identified by local Ebola alert numbers ${ }^{18}$ (online supplementary file 4).

In the routine context of Ethiopia, the verified events reported by CEBS for rabies, anthrax, acute flaccid paralysis and neonatal tetanus outnumbered the cases reported IBS during the same time in areas where CEBS was active, indicating better sensitivity of EBS compared with IBS $^{22}$ (online supplementary file 4 ).

\section{Acceptability}

In the context of the Ebola outbreak, Stone $e t a l^{17}$ evaluated acceptability as the proportion of CVs reporting for CEBS in Sierra Leone at least once per week; this increased from $69 \%$ to $93 \%$ during implementation. In addition, $74 \%$ of district stakeholders agreed that CEBS increased case detection and benefited their district through increased linkage with communities. ${ }^{17}$ CEBS was based on community health worker (CHW) and Red Cross volunteer networks that are largely known to the community prior to CEBS. ${ }^{16} 1719$

Santa-Olalla $e t a l^{25}$ reported that the near real-time information and response increased the acceptance by local authorities during the outbreak and willingness to institutionalise the system into the Ministry of Health immediately after the end of the outbreak in Haiti (online supplementary file 4).

In routine context, CEBS in Sierra Leone was well accepted by communities and CVs were appreciated, and CVs wanted to continue CEBS after the outbreak despite the lack of incentives. ${ }^{1516}$ Clara et $a l^{1314}$ reported "Key motivating factors for participation expressed by the VHWs (village health workers) were a sense of service to the community and opportunities to increase community ties, and improvement in community trust". Toyama $e t a l^{22}$ interpreted the proportion of rumours reported by the community to CEBS in Ethiopia as community acceptance: $30 \%$ of rumours were reported directly from the community (online supplementary file 4).

\section{Timeliness}

During the Ebola outbreak in Sierra Leone, the time from onset to detection for six confirmed Ebola cases in districts with active transmission was 1-3 days for 
four cases identified with CEBS and 5-7 days for two confirmed Ebola cases identified through other sources (online supplementary file 4). ${ }^{19}$

For the routine context in Vietnam, for EBS times from detection to notification at district level was reported to be $<24$ hours, mean time from detection to response was reported to be within 48 hours. ${ }^{14}$ The authors conclude that such a rapid response would not have been possible before the implementation of EBS. ${ }^{14}$ For CEBS in Ethiopia, a median time from onset to reporting of 3.8 days (95\% CI 2.2 to 5.3 days) was reported and from reporting to response of 0.6 days (95\% CI 0.1 to 1.2 days). ${ }^{22}$ In PNG's centralised routine EBS system, median time from onset to reporting was 10 days (range, 0-109 days) and median time from reporting to response was the same day $^{28}$ (online supplementary file 4).

\section{Flexibility and stability}

For the centralised EBS in Haiti and for CEBS in Sierra Leone, EBS was described as flexible to adapt and was broadened in scope, from EBS systems based on events in connection to one outbreak disease to generic EBS systems. ${ }^{15} 1625$

Sharma $e t a l^{27}$ described a successful shift in focus of the EBS system from generic EBS to influenza A (H1N1) specific events.

Tante et $a l^{23}$ described the stability of the routine EBS system in the Philippines after a natural disaster and found that $6 / 11$ affected areas had no interruptions of EBS operation, 3/11 areas were interrupted for 1 week while the two hardest hit areas had limited operationality for more than 7 weeks due to human resource and logistical limitations; $73 \%$ of all areas rated the EBS system as functional post-typhoon (online supplementary file 4).

\section{Cost estimates}

Several additional characteristics were evaluated (see online supplementary file 4), among them annual costs: data from Cambodia estimated the annual cost of CBS at US $\$ 0.5$ per capita for training, supervision and evaluation, including CEBS and the IBS and vital events component but excluding staff costs as all staff were previously in place ${ }^{26}$ For the Sierra Leone CEBS, total costs were estimated at US $\$ 1.3$ million start-up costs and US\$129000 monthly costs that covered training, telephones, motorbikes, fuel and incentives for 7416 CHWs and salaries for 137 surveillance supervisors ${ }^{19}$ (given a population of 3.9 million, $\sim \mathrm{US} \$ 0.4$ per capita annual costs).

\section{DISCUSSION}

\section{What are the strengths of EBS?}

The scope of EBS is broader than IBS in several aspects: (1) In outbreaks, EBS was able to identify outbreakdisease cases and programming gaps (eg, poor-quality cholera care) helping to guide the response $\mathrm{e}^{192025}$; (2) in outbreaks and routine context alike, EBS was able to identify outbreaks that were not detected by IBS, both for notifiable and non-notifiable diseases ${ }^{12-14} 192022$ 24-26 28 ; (3) in outbreaks and routine contexts, EBS was able to identify other potential hazards to human health such as natural events or animal die-offs ${ }^{12} 14222528$; (4) in outbreaks and routine context, EBS at the community level and open EBS were able to reach remote areas ${ }^{14-17} 192022252628$ and EBS was most used where health facilities and response was scarce in Haiti. ${ }^{25}$ In these settings, EBS provided an important channel for rapid reporting of events, of particular benefit to remote settings where IBS was poor. In addition, data from the Sierra Leone CEBS suggest that EBS systems were faster to identify cases from onset to detection ${ }^{19}$ than IBS and other CEBS systems were also reported to be faster than IBS. ${ }^{1422}$ However, across studies, no systematic and direct comparisons of timeliness of IBS and EBS were presented.

\section{What are the trade-offs for EBS?}

Many questions with regards to sensitivity and specificity of event definitions remain unanswered. For CEBS in outbreak settings, specified event definitions that indicate disease transmission had low specificity and event definitions were not always remembered long after trainings, ${ }^{17} 1920$ suggesting that definitions should be more rigorously tested prior to implementation. ${ }^{19}$ Telephone hotlines were mostly used for information by the public (and useful for improving communication with the public) but also identified cases that went under the radar of IBS ${ }^{18}$ and from areas outside the reach of IBS. ${ }^{25}$ EBS in communities showed a higher specificity than EBS using open hotlines, at the costs of limited reach (limited to communities with CEBS vs anywhere with phone connection). ${ }^{18} 19$ All EBS systems involved an increased workload for verification and assessment given the low specificity of the events and the volume of alerts produced. ${ }^{17-2125}$

In routine contexts, in which events were defined by a list of specific events (eg, for CEBS in Vietnam, Ghana and Sierra Leone), the main reported challenges included balancing sensitivity and specificity ${ }^{12}$ and uncertainties around event definitions. ${ }^{15}{ }^{16}$ In Vietnam, initially more than half of the reported events were endemic diseases that were routinely and rapidly reported through IBS, and therefore, event definitions that could indicate such diseases were subsequently removed. ${ }^{13}$ Balancing sensitivity against specificity is particularly challenging as there is no gold standard for detection of events and non-events that would allow determination of sensitivity and specificity and available evidence is anecdotal, that is, reports of outbreaks identified by EBS but not IBS. ${ }^{22}$ In addition, balance of sensitivity versus specificity will inevitably depend on the setting, existing surveillance, access to healthcare and whether an outbreak is ongoing. CEBS systems that were based on undefined events such as 'unusual events' showed a PPV $>0.6{ }^{22}{ }^{26}$ The open EBS system in PNG notifying 'public health events' also exhibited a PPV $>0.8$, possibly because it was mainly used by local public health authorities to notify to central level. ${ }^{28}$ There seems to be some indication that the PPV of undefined events is not worse than the PPV of defined events pointing to specific diseases; however, we do 
not know if this comes at the costs of lower sensitivity to detect outbreaks.

The amount of work for verification and response is higher in EBS systems than IBS systems, as every notified signal requires rapid verification and potentially response. In outbreak settings, all EBS were set up with international support ${ }^{17-21}{ }^{25}$; in routine contexts, dedicated national government leadership was often paired with regional or international support to sustain verification and response systems. ${ }^{12-16}$ 22-24 26-28

With regard to costs, while most reports did not account for total direct costs, ${ }^{19}{ }^{26}$ costs structures would be influenced by the provision of additional capacity for verification of the potentially large number of signals produced by EBS. Where human resources and response elements are not already in place, costs might be associated with EBS, that not currently absorbed by the surveillance system's budget (ie, incentives for CHWs, additional verification capacity, including rapid response teams etc).

\section{How to implement it?}

In outbreak and non-outbreak settings, early engagement and meetings with all stakeholders (including community leaders) were described as crucial ${ }^{14171920}$ to ensure ownership, increase coordination and gain "better understanding of the existing surveillance landscape". ${ }^{14}$

With regards to surveillance, Santa-Olalla et $a t^{25}$ described the importance of not undermining existing responsibilities and capacities for EBS cholera surveillance in Haiti. Routine context CEBS in Vietnam was developed at ministry of health level with involvement of all stakeholders to ensure integration into existing surveillance infrastructure. ${ }^{14}$ In Cambodia and Ethiopia, CEBS was reported to be functional mainly because it was simple and built on the existing surveillance systems and resources. ${ }^{22} 26$

Good linkage to response was described as essential for all systems as every verified event needs an assessment and potentially response. ${ }^{8-24}$ However, response was challenging in some systems and required scaling up. ${ }^{21} 222528$ All outbreak-specific EBS systems were linked to pre-existing response structures set up specifically for the outbreak. ${ }^{17-21}{ }^{25}$ In order to ensure timely reporting of defined events and sustainability, stakeholders in EBS need training and, if possible, monitoring, supervision and refresher trainings. ${ }^{14-17} 2022$ Several reports suggested that standardised documentation and assessment improved accountability and ensured a consistent approach. ${ }^{1422} 28$ In routine contexts, where the capacity to conduct risk assessment and field investigations is low, specific resources and training are required. ${ }^{1428}$

Several EBS systems incorporated feedback mechanisms to stakeholders to inform about findings and actions; all records that reported such feedback loops identified them as essential instruments to sustain motivation, especially in routine settings, and to improve cooperation. ${ }^{14252628}$ Communication improved between actors in the surveillance system and the community ${ }^{26}$ but also between human and animal health ${ }^{14}$ and stakeholders at different levels at the surveillance system. ${ }^{28}$

\section{Limitations}

There are some limitations stemming from the variety of EBS systems as well as evaluation designs and the poor evidence rating of the studies. The number of studies with low-quality evidence grading is due to the nature of surveillance systems evaluations which are inherently descriptive and seldom have a comparator. All studies were of low evidence and none compared EBS and IBS systems systematically, controlled or randomised. Most importantly, surveillance attributes, especially PPV, were not always reported or analysed in the same way, complicating meaningful comparisons. For the PPV, information on if and how signals that did not result in alerts were documented was frequently lacking.

Additionally, the number of studies was low, and more descriptions and rigorous evaluations are therefore needed to generate more conclusive evidence. Despite the increasing focus on EBS as a central component for early detection of outbreaks, there is limited guidance on EBS evaluation. The development of guidance more tailored to the evaluation of EBS would be useful as would be planning and publishing evaluations of EBS systems more systematically to ensure lessons learnt are documented and communicated.

Furthermore, all EBS systems succeeded in identifying events and outbreaks; however, we cannot rule out that publication bias towards successful systems is the reason for that finding.

In addition, other countries may have EBS systems in place, but their description might not be included in the paper given the absence of literature on them.

\section{CONCLUSION}

Health facility and community-based EBS provide valuable information that can strengthen the early warning function of national surveillance systems. Every signal generated by EBS requires verification, and each EBS system is only as good as the capacity to respond to verified events to ensure that EBS can lead to meaningful public health action. ${ }^{8-24}$ This requires close integration with existing surveillance systems and this needs to happen in a way that does not undermine existing responsibilities and capacities. ${ }^{10} 12-17192022232528$ EBS can be a powerful tool to improve rapid communication between actors in healthcare and surveillance, and feedback loops to all stakeholders including communities were described as essential instruments for sustaining motivation and cooperation. ${ }^{16171920232528}$ Where EBS is not a fully integrated function of a surveillance system, priority in its implementation should be given to settings that would particularly benefit from EBS strengths. This includes areas most prone to outbreaks, where otherwise undetected health 
threats will most likely occur, where access to the formal health system is limited and where traditional 'routine' surveillance is suboptimal.

Acknowledgements AK would like to thank the team of the UK Public Health Rapid Support Team for their support throughout study development and manuscript preparation. The authors wish to thank Philip Abdelmalik for his thorough review of the manuscript.

Contributors AK designed the study and developed the search strategy. PK, JP, $\mathrm{CH}, \mathrm{OLPdW}$ and RR provided feedback on search strategy and study design. AK conducted the systematic literature search. AK, PK, OLPdW, RR and JP reached out to partners for grey literature. AK and PK conducted the review of literature, abstracts and full texts. AK conducted data extraction and quality assessment, performed the content analysis, wrote the manuscript, and prepared all tables and figures. PK contributed to data extraction and quality assessment. RR provided input to the surveillance system evaluation and content and structure of the manuscript. PK, JP, CH, KS, OLPdW and RR reviewed and approved the final manuscript. RR conducted the proofreading of the final manuscript.

Funding The UK Public Health Rapid Support Team is funded by the National Institute for Health Research and Department of Health and Social Care.

Disclaimer The views expressed are those of the authors and not necessarily those of the NIHR or DHSC. The authors alone are responsible for the views expressed in this article and they do not necessarily represent the views, decisions or policies of the institutions with which they are affiliated.

Competing interests None declared.

Patient consent for publication Not required.

Provenance and peer review Not commissioned; externally peer reviewed.

Data availability statement There are no data in this work. All data relevant to the study are included in the article or uploaded as online supplementary information.

Open access This is an open access article distributed in accordance with the Creative Commons Attribution Non Commercial (CC BY-NC 4.0) license, which permits others to distribute, remix, adapt, build upon this work non-commercially, and license their derivative works on different terms, provided the original work is properly cited, appropriate credit is given, any changes made indicated, and the use is non-commercial. See: http://creativecommons.org/licenses/by-nc/4.0/.

\section{ORCID iDs}

Anna Kuehne http://orcid.org/0000-0002-8578-0362

Patrick Keating https://orcid.org/0000-0003-3358-3196

Jonathan Polonsky https://orcid.org/0000-0002-8634-4255

Christopher Haskew https://orcid.org/0000-0003-1992-808X

Karl Schenkel https://orcid.org/0000-0001-5444-9143

Olivier Le Polain de Waroux https://orcid.org/0000-0003-3458-2945

Ruwan Ratnayake http://orcid.org/0000-0003-4978-6668

\section{REFERENCES}

1 WHO. International health regulations, 2005.

2 WHO. Early detection, assessment and response to acute public health events: implementation of early warning and response with a focus on event-based surveillance. interim version, 2014.

3 WHO Western Pacific Region. A guide to establishing event-based surveillance, 2008. Available: http://www.wpro.who.int/emerging_ diseases/documents/docs/eventbasedsurv.pdf

4 WHO. Outbreak surveillance and response in humanitarian emergencies. WHO guidelines for EWARN implementation, 2012. Available: http://www.who.int/diseasecontrol_emergencies/ publications/who_hse_epr_dce_2012.1/en/index.html

5 IFRC. Community-based surveillance guidance, 2016.

6 IFRC SLRC. Project Description and Standard Operating Procedure for Community Event-based surveillance (CEBS)-adapted from the Ebola Response Consortium's Standard Operating Procedure for Community Event Based Surveillance for Ebola Virus Disease in Sierra Leone, 2015.

7 The World Bank Group. New country classifications by income level: 2017-2018, 2017. Available: http://blogs.worldbank.org/opendata/ new-country-classifications-income-level-2017-2018
8 Schünemann H, Brozek J, Guyatt G, et al. GRADE handbook for grading quality of evidence and strength of recommendations [Internet]. The GRADE Working Group, 2013. Available: https://gdt. gradepro.org/app/handbook/handbook.html

9 German RR, Lee LM, Horan JM, et al. Updated guidelines for evaluating public health surveillance systems: recommendations from the guidelines Working group. MMWR Recomm Rep 2001;50:1-7.

10 Smith J, Firth J. Qualitative data analysis: the framework approach. Nurse Res 2011;18:52-62.

11 Technical Contributors to the June WHO Meeting. A definition for community-based surveillance and a way forward: results of the WHO global technical meeting, France, 26 to 28 June 2018. Euro Surveill 2019;24.

12 Merali S, Asiedu-Bekoe F, Adjabeng M. Lessons learned from community event-based surveillance implementation in Ghana. Atlanta: ICEID, 2018.

13 Clara A, Dao ATP, Do TT, et al. Factors influencing community eventbased surveillance: lessons learned from pilot implementation in Vietnam. Health Security 2018;16:S-66--60.

14 Clara A, Do TT, Dao ATP, et al. Event-based surveillance at community and healthcare facilities, Vietnam, 2016-2017. Emerg Infect Dis 2018;24:1649-58.

15 Larsen TM, Brux Mburu C, Kongelf A, et al. Red Cross volunteers' experience with a mobile community event-based surveillance (CEBS) system in Sierra Leone during- and after the Ebola outbreak - a qualitative study. Heal Prim Car 2016;1:1-7.

16 Larsen TM, Brux Mburu C, McClelland AL, et al. A qualitative study of volunteer experiences with a mobile community event- based surveillance (CEBS) system in Sierra Leone. Int $J$ Infect Dis 2016.

17 Stone E, Miller L, Jasperse J, et al. Community event-based surveillance for Ebola virus disease in Sierra Leone: implementation of a national-level system during a crisis. PLoS Curr 2016;8. doi:10.1371/ currents. outbreaks.d119c71125b5cce312b9700d744c56d8. [Epub ahead of print: 07 Dec 2016]

18 Lee CT, Bulterys M, Martel, LD, et al. Evaluation of a national call center and a local alerts system for detection of new cases of Ebola virus disease-Guinea, 2014-2015. MMWR Morb Mortal Wkly Rep 2016;65:227-30.

19 Ratnayake R, Crowe SJ, Jasperse J, et al. Assessment of community event-based surveillance for Ebola virus disease, Sierra Leone, 2015. Emerg Infect Dis 2016;22:1431-7.

20 Ebola Response Consortium. Evaluation and functionality and effectiveness of community event-based surveillance (CEBS) in Sierra Leone, 2015.

21 Miller LA, Stanger E, Senesi RG, et al. Use of a nationwide call center for Ebola response and monitoring during a 3-day houseto-house campaign - Sierra Leone, September 2014. MMWR Morb Mortal Wkly Rep 2015;64:28-9.

22 Toyama Y, Ota M, Beyene BB. Event-based surveillance in northwestern Ethiopia: experience and lessons learnt in the field. Western Pac Surveill Response J 2015;6:22-7.

23 Tante S, Villa E, Pacho A, et al. Which surveillance systems were operational after Typhoon Haiyan? Western Pac Surveill Response J 2015;6(Suppl 1):66-70.

24 Naser AM, Hossain MJ, Sazzad HMS, et al. Integrated cluster- and case-based surveillance for detecting stage III zoonotic pathogens: an example of Nipah virus surveillance in Bangladesh. Epidemiol Infect 2015;143:1922-30.

25 Santa-Olalla P, Gayer M, Magloire R, et al. Implementation of an alert and response system in Haiti during the early stage of the response to the cholera epidemic. Am J Trop Med Hyg 2013;89:688-97.

26 Oum S, Chandramohan D, Cairncross S. Community-based surveillance: a pilot study from rural Cambodia. Trop Med Int Health 2005;10:689-97.

27 Sharma R, Ratnesh L, Karad AB, et al. Communicable disease outbreak detection by using supplementary tools to conventional surveillance methods under integrated disease surveillance project (IDSP), India. J Commun Dis 2009;41:149-59.

28 Dagina R, Murhekar M, Rosewell A, et al. Event-based surveillance in Papua New Guinea: strengthening an international health regulations (2005) core capacity. Western Pac Surveill Response J 2013;4:19-25.

29 AbdelMalik P, Peron E, Schnitzler J, et al. The epidemic intelligence from open sources initiative: a collaboration to harmonize and standardize early detection and epidemic intelligence among public health organizations. Wkly Epidemiol Rec 2018;20:267-9.

30 Mykhalovskiy E, Weir L. The global public health intelligence network and early warning outbreak detection: a Canadian contribution to global public health. Can J Public Health 2006;97:42-4. 